\title{
Modification of Glycan Expression in Staphylococcus epidermidis to Prevent Biofilm Formation
}

\author{
Ekpiken Solomon Ekpiken*, Lawrence B. Etim and Mercy Ekong \\ Department of Biological Sciences Cross River University of Technology, Nigeria \\ *Corresponding author
}

\section{A B S T R A C T}

Staphylococcus epidermidis is a common commensal of the skin and mucous membranes of humans. However, it is a common cause of nosocomial infection especially infection associated with the insertion of biomedical devices. Glycans have

\section{Keywords}

Glycosylation inhibitors, Staphylococcus epidermidis, Desialylation; Glycans; enzymelinked lectin assay (ELLA) and Lectins.

\section{Article Info}

Accepted: 10 July 2017 Available Online: 10 September been shown to mediate vast majority of biological processes and its alterations have been reported to be used as biomarkers and applied in diagnosis of malignancies and infections. The absolute mean Optical Density $\left(\mathrm{OD}_{450 \mathrm{~nm})}\right.$ of the biofilm was calculated as $0.248 \pm 0.044 \mathrm{SD}$ and the biofilm was observed to strongly adhere to the microtitre plate surface. The biofilm growth curve peaks at $2 \mathrm{hrs}$ with a mean $\mathrm{OD}_{450 \mathrm{~nm}}$ of 0.385 ; at 30 minutes and $4 \mathrm{hrs}$ growth, mean $\mathrm{OD}_{450 \mathrm{~nm}}$ were 0.365 and 0.383 respectively and drastically drops to 0.248 at $24 \mathrm{hrs}$. A panel of 11 biotinylated lectins was used to detect any alterations in glycosylation of the glycans expressed by of S. epidermidis RP62A (ATCC 35984). Glycans' expression varies with time and most glycans showed strong expression at $2 \mathrm{hrs}$ of incubation except $\mathrm{N}$-acetyl galactosamine (GalNAc), which showed strong expression at 30 minutes. Glycosylation inhibitors such as Neuraminidase, Cytidine 5'- monophospho-N- acetylneuraminic acid sodium salt (CMP-NANA), Sialic acid aldolase and 1M Hydrochloric acid were used to prevent biofilm establishment and development and their effects evaluated using toluidine blue stain and lectin screening. The glycosylation inhibitors showed remarkable 80\% reduction in Extracellular Polysaccharides (EPS) production and the expression of glycans in comparison with the control, and were most effective at $2 \mathrm{hrs}$ relative to $0 \mathrm{hr}$ and $4 \mathrm{hrs}$ of incubation except $1 \mathrm{M} \mathrm{HCl}$, which was effective at $0 \mathrm{hr}$ compared to other times of incubation. This study reveals new strategies of using glycosylation inhibitors to prevent biofilm formation during implant of medical

\section{Introduction}

Biofilms are complex multicellular microbial communities immobilised by extracellular polysaccharides (EPS) that is produced by microorganisms, and can be attached to biotic and abiotic surfaces (Otto, 2009). It occurs in response to adverse environmental condition or stress and involves multiple regulatory signals that reprogramme gene expression (Monds and O'Toole
2009). This gene expression reprogramming alters the expression of virulence factors, nutrient utilisation, surface molecules and equips the bacteria with the arsenals which enable their survival in adverse environmental conditions (Klebensberger et al., 2009). Đapa et al. (2013) showed that lower concentrations of antibiotics can cause stress in bacteria thereby inducing biofilm formation. The structure of EPS keeps bacteria in close proximity, which enables them to 
interact closely and exchange genetic materials (Flemming and Wingender, 2010; Koo, et al., 2010) The intimate cell-to-cell interactions promote the spread of antibiotic resistance genes and other virulence factors among bacteria within the biofilm (Vuong et al., 2004).

Medical device infections constitute a major cause of morbidity and mortality in hospitalised patients, and biofilms readily develop on all types of medical devices inserted in more than $25 \%$ of hospitalised patients (Hazan et al., 2006). Approximately $60-70 \%$ of nosocomial infections are associated with implanted medical devices (Bryers, 2008). Catheter associated urinary tract infections account for $43 \%$ of nosocomial infections in UK (Hopkins et al., 2011). Bacterial infections in patients with urinary catheters occur at $5-10 \%$ per day in patients with long term catheters ( $\geq 28$ days) (Maki and Tambyah 2001).

S. epidermidis infections rarely develop into life threatening diseases, however, they are extremely difficult to treat and recurrently posing a serious public health burden (Otto, 2009). Although $S$. epidermidis is a normal flora of the skin, it is a common cause of nosocomial infection especially infections associated with the implant of medical devices such as urinary catheters (Cogen et al., 2008).

Lectins are carbohydrate binding proteins that can recognise and bind to specific glycan molecules (Sharon and Lis 2002). Altered glycan molecules have been reported as indicators for the development of malignant cells (Rosenfeld et al., 2007); and can be applied as biomarkers for diagnosis of infections (Chen et al., 2007).

Previous study had focused on the inhibition of bacterial attachment or colonisation on implanted medical devices like urinary catheters, by using physiochemical modifications on the device surface to create anti- adhesive surfaces; coating catheters with antimicrobial agents, silver salts (Hazan et al., 2006), however, these approaches provide minimal effort in reducing biofilm associated infection. Glycan modification occurs during the later stages of biofilm development and sialylation of glycan molecules are involved in biofilm establishment. This research therefore focuses on the desialylation of these glycan molecules by treating with specific glycosylation inhibitors to prevent biofilm formation and development.

\section{Materials and Methods}

\section{Materials}

S. epidermidis RP62A (ATCC 35984) cultured in blood agar plate (Oxoid, England) was subcultured in $10 \mathrm{ml}$ tryptone soy broth (TSB) (Oxoid, England) and incubated for $24 \mathrm{hrs}$ at $37^{\circ} \mathrm{C}$.

\section{Biofilm growth in a microtitre plate}

S. epidermidis biofilms were grown according to the procedure described by Deighton et al. (2001) with slight modifications. Approximately $50 \mu 1$ of fresh TSB culture containing $3.0 \times 10^{6} \mathrm{cfu} / \mathrm{ml}$ was inoculated into 8 wells of a flat bottomed microtitre plate (Greiner bio-one, Germany) and incubated for $24 \mathrm{hrs}$ at $37^{\circ} \mathrm{C}$. After incubation, excess medium containing planktonic cells were removed and the plate washed with $1 \times$ Trisbuffered saline (TBS, pH 7.6) for three times using ELISA washer (bioTek, USA) and dried for at least $2 \mathrm{hrs}$ at room temperature. The optical density $\left(\mathrm{OD}_{450 \mathrm{~nm}}\right)$ was measured using a microplate reader (Titertek Multiskan MCC/340, Finland).

\section{Crystal violet staining and time-course analysis}

S. epidermidis biofilms were grown according to the procedure described by Deighton et al. (2001) with slight modifications and incubated for 30 minutes, $2 \mathrm{hrs}$, $4 \mathrm{hrs}$ and $24 \mathrm{hrs}$ at $37^{\circ} \mathrm{C}$ respectively. The microtitre plates were stained using $0.4 \%$ (w/v) ammonium crystal violet stain and incubated for 10 minutes at room temperature to evaluate the presence of bacterial cells in the biofilms. Excess stain was removed and the plates rinsed twice with tap water and dried for at least $2 \mathrm{hrs}$ at room temperature, and the $\mathrm{OD}_{450 \mathrm{~nm}}$ was measured using a microplate reader (Titertek Multiskan MCC/340, Finland).

\section{Lectins screening and time-course analysis}

Approximately 50 $\mu 1$ of fresh TSB culture containing $3.0 \times 10^{6} \mathrm{cfu} / \mathrm{ml}$ was inoculated into each 
well of 4 microplates and incubated for 30 minutes, $2 \mathrm{hrs}$, $4 \mathrm{hrs}$ and $2 \mathrm{hrs}$ at $37^{\circ} \mathrm{C}$ respectively. Planktonic cells were discarded in a disinfectant and the plates dried for at least $2 \mathrm{hrs}$ at room temperature. Enzyme-linked lectin assay (ELLA) was performed according to manufacturers' instructions using a panel of 11 different biotinylated lectins (Vector Laboratories), with different specificity which include: Sambucus nigra agglutinin (SNA), Wheatgerm agglutinin (WGA), succinylated wheatgerm agglutinin (sWGA), Galanthus nivalis agglutinin (GNA), Maackia amurensis agglutinin II (MAA- II), Soybean agglutinin (SBA), Solanum tuberosum agglutinin (STA), Erythrina cristagalli agglutinin (ECA), Lens culinaris agglutinin (LCA), LPhaseolus vulgaris (IPHA) and Concanavalin A (ConA). This analysis was performed in triplicate. Wells without biotinylated lectin served as negative control while wells without biofilm served as blank wells. The $\mathrm{OD}_{450 \mathrm{~nm}}$ of each well was measured using a microplate reader.

\section{Biofilm time-dependent inhibition Assay}

Approximately $50 \mu \mathrm{l}$ of fresh TSB culture containing $3.0 \times 10^{6} \mathrm{cfu} / \mathrm{ml}$ was inoculated into each well of 4 microplates and $1 \mu \mathrm{l}$ each of Neuraminidase (from Clostridium perfringens) (1U/ml), CMP-NANA (cytidine 5'monophospho-N- acetylneuraminic acid sodium salt) $(100 \mu \mathrm{g} / \mathrm{ml})$, Sialic acid aldolase (from E. coli $\mathrm{K}-12)(200 \mu \mathrm{g} / \mathrm{ml})$ (Sigma Life Science) and 1M Hydrochloric acid $(\mathrm{HCl})$ were added to each plate at $0 \mathrm{hr}, 2 \mathrm{hrs}$ and $4 \mathrm{hrs}$ respectively; and incubated for $24 \mathrm{hrs}$ at $37^{\circ} \mathrm{C}$. After incubation, the plates were washed with 1xTris- buffered saline (TBS, $\mathrm{pH}$ 7.6) thrice using ELISA washer and dried for at least $2 \mathrm{hrs}$ at room temperature. Enzyme-linked lectin assay (ELLA) was repeated according to manufacturer's instruction using 6 different biotinylated lectins including SNA, WGA, sWGA, GNA, ECA and ConA. The lectins discontinued were those with similar glycan specificity with the ones selected and those that showed no obvious interaction with the glycans, therefore they would provide no additional information. This analysis was performed in triplicate. Wells without the treatment served as the positive control while wells without biofilm served as the negative control. The $\mathrm{OD}_{414 \mathrm{~nm}}$ of each well was measured using a microplate reader.

\section{Toluidine blue staining}

The effect of the treatments to prevent biofilm development at different incubation times was evaluated using $0.25 \%(\mathrm{w} / \mathrm{v})$ toluidine blue stain and incubated for 30 minutes at room temperature. The $\mathrm{OD}_{450 \mathrm{~nm}}$ of each well was measured using a microplate reader.

\section{Results and Discussion}

\section{Biofilm growth in a microtitre plate}

The detection of in vitro biofilm formation of $S$. epidermidis RP62A (ATCC 35984) in a microtitre plate after 24 hours of incubation was evaluated using crystal violet stain, which is usually used as the gold standard. The absolute mean $\mathrm{OD}_{450 \mathrm{~nm}}$ of the biofilm was calculated as $0.248 \pm 0.044 \mathrm{SD}$ and the biofilm was observed to strongly adhere to the microtitre plate surface.

\section{Biofilm growth and time-course analysis}

S. epidermidis biofilm growth curve stained with crystal violet after $24 \mathrm{hrs}$ incubation is presented in figure 1. The biofilm growth curve shows a downward curve due to EPS formation. This result indicates that $S$. epidermidis grows optimally at $2 \mathrm{hrs}$ and biofilm develops and decreases at $4 \mathrm{hrs}$ due to EPS formation. The growth curve peaks at $2 \mathrm{hrs}$ with a mean $\mathrm{OD}_{450 \mathrm{~nm}}$ of 0.385 ; at 30 minutes and $4 \mathrm{hrs}$ growth mean $\mathrm{OD}_{450 \mathrm{~nm}}$ were 0.365 and 0.383 respectively and drastically drops to 0.248 at 24hrs.

\section{Lectins screening and time-course analysis}

A panel of lectins was screened at different time as shown in Figure 2. The selection of the lectins was based on the degree of specificity with glycans expressed during biofilm formation. Eleven lectins were analysed after 30 minutes, $2 \mathrm{hrs}$, $4 \mathrm{hrs}$ and $24 \mathrm{hrs}$ of incubation. At 30 minutes of incubation, it was observed that $\mathrm{N}$ - acetyl glucosamine (GlcNAc) binding lectins: STA and sWGA showed very strong interaction with the sugar, GlcNAc. It was equally observed that $\mathrm{N}$ - acetyl galactosamine (GalNAc) binding lectin, SBA 
showed strong interaction with terminal $\alpha$ GalNAc. However, GNA and ConA were observed to have strong interaction with mannose than LCA. At $2 \mathrm{hrs}$ of incubation, mannose was strongly expressed and interacted with GNA, LCA and ConA, Also, sialic acid was strongly expressed and interacted with SNA and WGA (which also interact with GlcNAc); terminal sialic acid attached to $\alpha-2,3 \mathrm{Gal}$ or on $\mathrm{N}$-glycan cores was equally expressed and interacted with MAAII. However, STA and sWGA interacted with GlcNAc and was also expressed along with galactose which interacted with ECA. At $4 \mathrm{hrs}$ it was observed that SNA and WGA showed strong interaction with sialic acid and sWGA also interacted with GlcNAc while other sugars were either masked or modified, particularly mannose, galactose and $\mathrm{N}$ - acetyl galactosamine. At $24 \mathrm{hrs}$ of incubation, sialic acid, mannose and GalNAc were expressed and interacted with SNA, WGA, GNA and SBA respectively. Galactose and GlcNAc were also expressed and interacted with ECA, STA and sWGA. Other glycans like terminal mannose and complex glycan structure were either masked or modified and showed weak interaction with their lectins.

\section{Biofilm time-dependent inhibition assay}

The glycosylation inhibitors used include neuraminidase, CMP-NANA, sialic acid aldolase and hydrochloric acid $(\mathrm{HCl})$. The effect of neuraminidase on EPS production in $S$. epidermidis biofilm development at different incubation times was analysed using toluidine blue stain as shown in figure 3 a. There was a $90 \%$ reduction in EPS production when the biofilm was treated with neuraminidase in comparison with the control. However, treatment with neuraminidase was most effective at $2 \mathrm{hrs}$ of treatment in comparison with $0 \mathrm{hr}$ and $4 \mathrm{hrs}$. Also, the effect of neuraminidase on sialylation in $S$. epidermidis biofilm development at different incubation times was analysed using lectin staining as shown in figure $3 \mathrm{~b}$. There was a remarkable reduction in glycan expression compared to the control. Neuraminidase cleavage was most effective at $0 \mathrm{hr}$ and $2 \mathrm{hrs}$, compared to $4 \mathrm{hrs}$ of treatment so a weak and decreased interaction with SNA and WGA was observed. Also, GlcNAc expression was reduced at $0 \mathrm{hr}$ and $2 \mathrm{hrs}$ in comparison to $4 \mathrm{hrs}$ of incubation. Generally speaking, sialic acid cleavage dramatically influenced the expression of other glycans. High mannose reduction was most effective at $2 \mathrm{hrs}$ compared to $0 \mathrm{hr}$ and $4 \mathrm{hrs}$ as shown with GNA interaction. Galactose and terminal mannose reduction were most effective at Ohr and $2 \mathrm{hrs}$ compared to $4 \mathrm{hrs}$ as shown with ECA interaction; and $2 \mathrm{hrs}$ and $4 \mathrm{hrs}$ compared to $0 \mathrm{hr}$ as shown with ConA interaction respectively.

The effect of CMP-NANA on EPS production in S. epidermidis biofilm development at different incubation time was analysed using toluidine blue stain and presented in figure $4 \mathrm{a}$. There was a remarkable reduction in EPS production when treated with CMP-NANA in comparison to the control; however, treatment was most effective at $2 \mathrm{hrs}$ compared with $0 \mathrm{hrs}$ and $4 \mathrm{hrs}$. Figure $4 \mathrm{~b}$ shows the effect of CMP-NANA on sialylation of $S$. epidermidis biofilm development at different incubation time analysed using lectin staining. It was observed that CMP-NANA treatment remarkably reduced sialylation as compared to the control. Overall effectiveness of CMP-NANA was observed at $0 \mathrm{hr}$ except in galactose, which was effective at $4 \mathrm{hrs}$. Sialic acid reduction was observed to be effective at $0 \mathrm{hr}$ though not remarkable as compared to $2 \mathrm{hrs}$ and $4 \mathrm{hrs}$ as observed with SNA and WGA interactions. Galactose and GlcNAc reduction was also observed most effective at $4 \mathrm{hrs}$ and $0 \mathrm{hr}$ as observed with ECA and sWGA interaction respectively. However, mannose was reduced at Ohr and 4hrs as observed with GNA and ConA interaction.

The effect of sialic acid aldolase on EPS production in S. epidermidis biofilm development was analysed at different incubation time using toluidine blue stain and is shown in figure 5a. A dramatic reduction in EPS production was observed with sialic acid aldolase treatment as compared with the control. A remarkable reduction was observed at $2 \mathrm{hrs}$ in comparison with $0 \mathrm{hr}$ and $4 \mathrm{hrs}$ due to degradation of sialic acid into its component parts. The effect of the treatment of $S$. epidermidis biofilm with sialic acid aldolase was analysed using lectin staining and is shown in figure $5 \mathrm{~b}$; a remarkable reduction in the expression of glycans as compared to the control was observed. Sialic acid reduction was effective at $0 \mathrm{hr}$ 
relative to $2 \mathrm{hrs}$ and $4 \mathrm{hrs}$ as detected by SNA, However, both sialic acid and GlcNAc expression showed dramatic reduction over time as detected by WGA. GlcNAc expression was also reduced and effective at $2 \mathrm{hrs}$ as detected by sWGA. Galactose reduction was effective at $0 \mathrm{hr}$; however mannose reduction was effective at $0 \mathrm{hr}$ and $4 \mathrm{hrs}$ as observed with ConA and GNA.

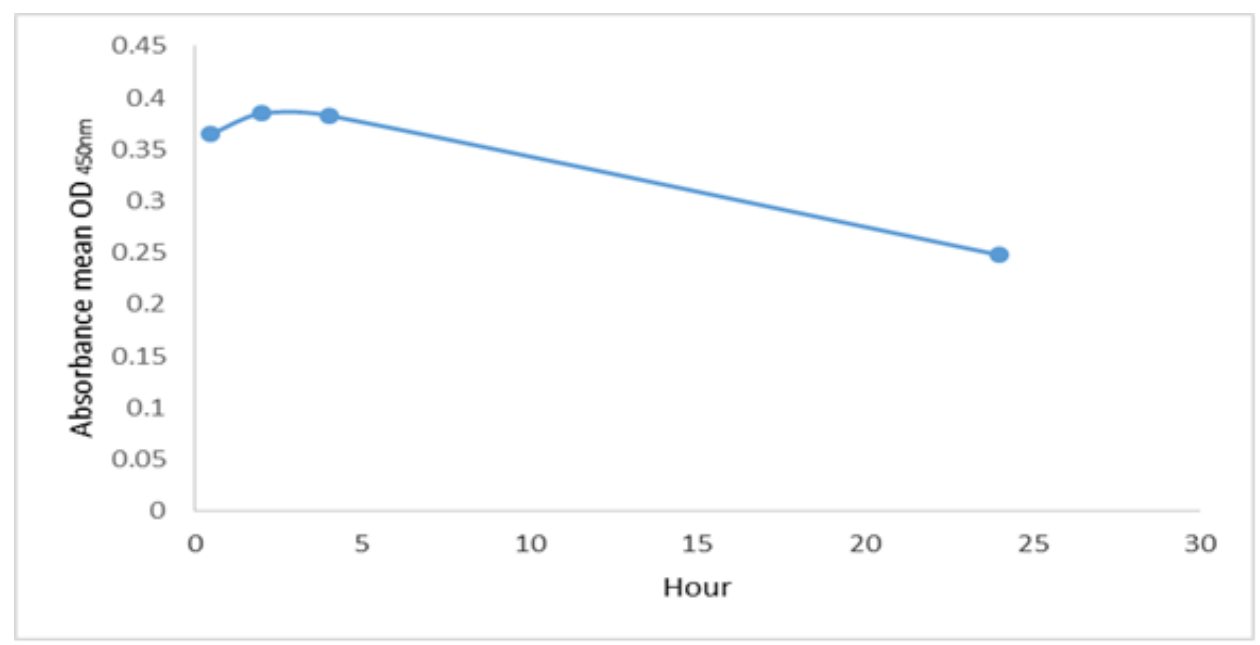

Figure 1: Biofilm growth curve

Cnystal violet stain of a biofilm grouth at 30 minutes, $2 \mathrm{hrs}, 4 \mathrm{hrs}$ and $24 \mathrm{hrs}$. The curve peaks at $2 \mathrm{hrs}$ and start decreasing. This result indicates that the S. epidernidis RP62A grows optimally at $2 \mathrm{hrs}$ and as EPS start forming, bacterial cells were not detected by the stain as they were
enveloped in the EPS.

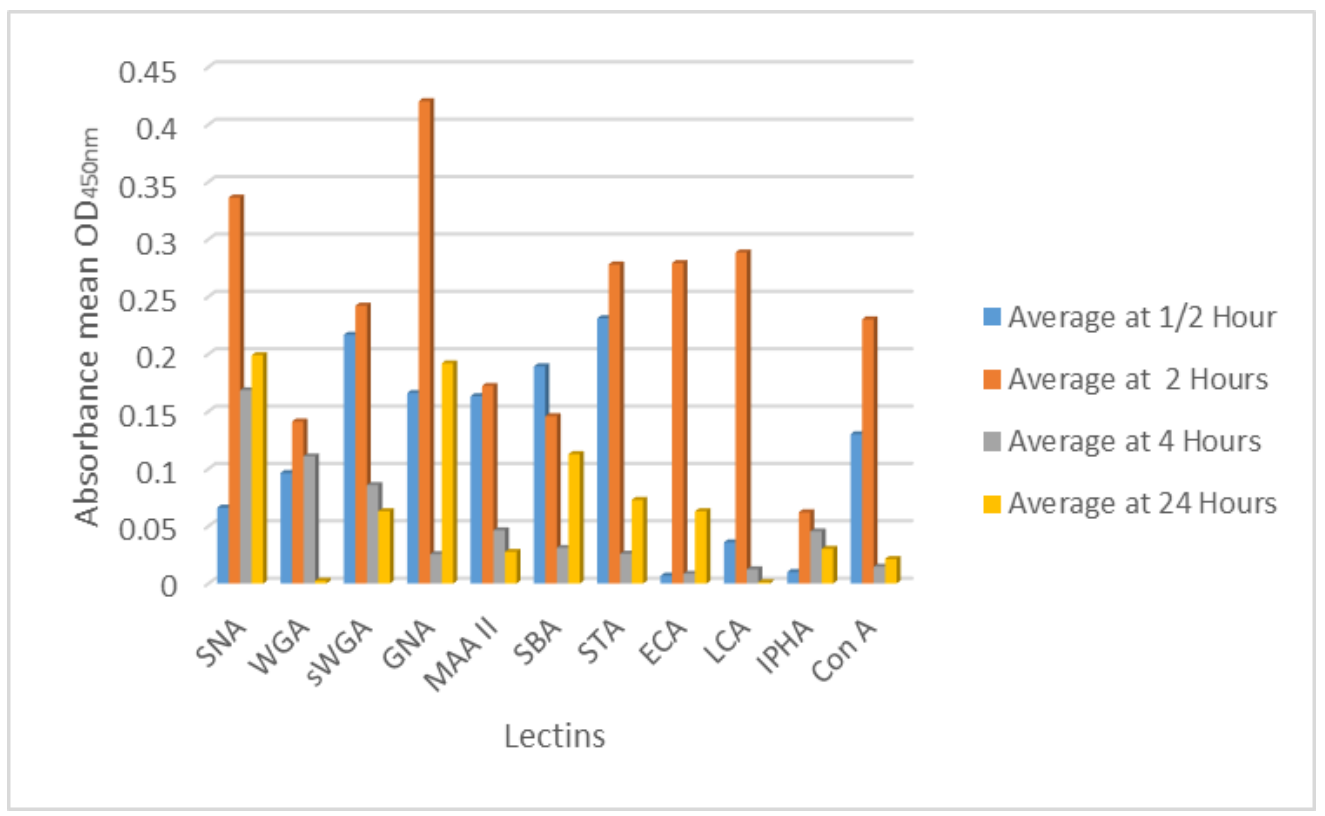

Figure.2 Lectin screening at different time-course

Most of the glycans were observed to be mostly expressed at $2 \mathrm{hrs}$, however, GlcNAc, mannose, terminal sialic acid $\alpha-2,3 \mathrm{Gal}$, terminal $\alpha$-GalNAc were expressed at 30 minutes though Gal $\beta$ 1,4 GlcNAc and highly branched non-bisected complex N-glycans were not expressed at this time. Those glycans that were not expressed were either masked or modified as such the lectins cannot bind. 

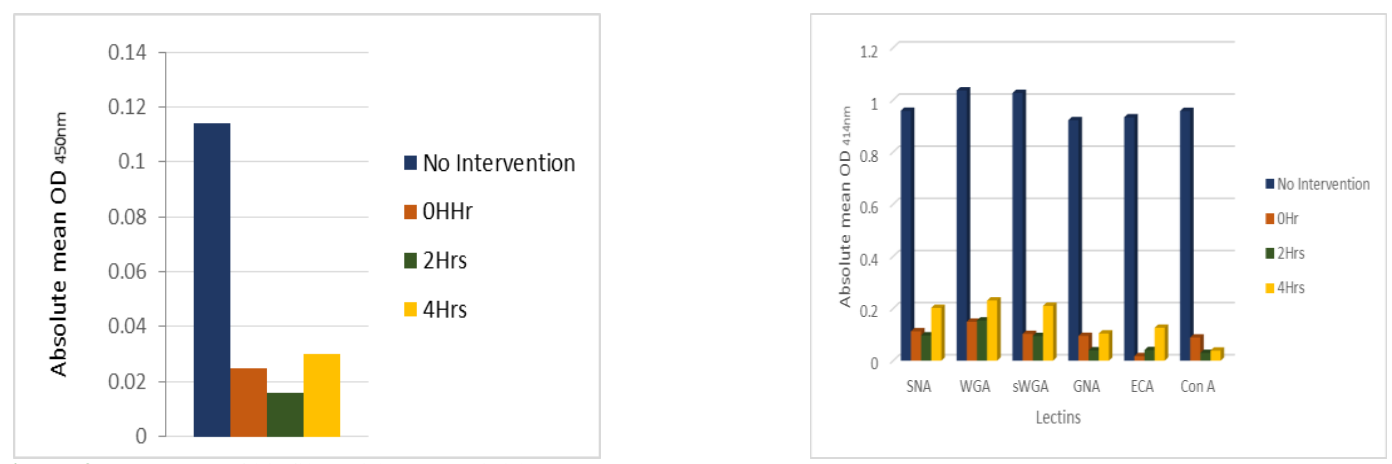

Figure.3 Treatment of biofilm with neuraminidase

(a) The effect of neuraminidase on EPS at different incubation time stained with toluidine blue. (b) The effect of neuraminidase on EPS at different incubation times with lectin staining. A dramatic decrease in the expression of the glycans
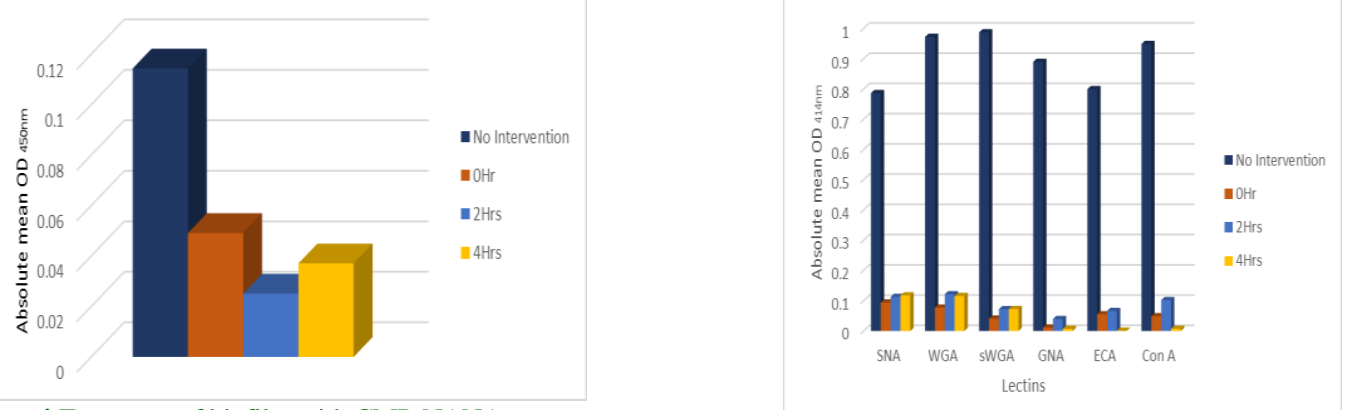

Figure.4 Treatment of biofilm with CMP-NANA

(a) The effect of NANA in EPS production to prevent biofilm development at different incubation times. (b) The effect of CMP-NANA on sialylation at different incubation times with lectin staining.
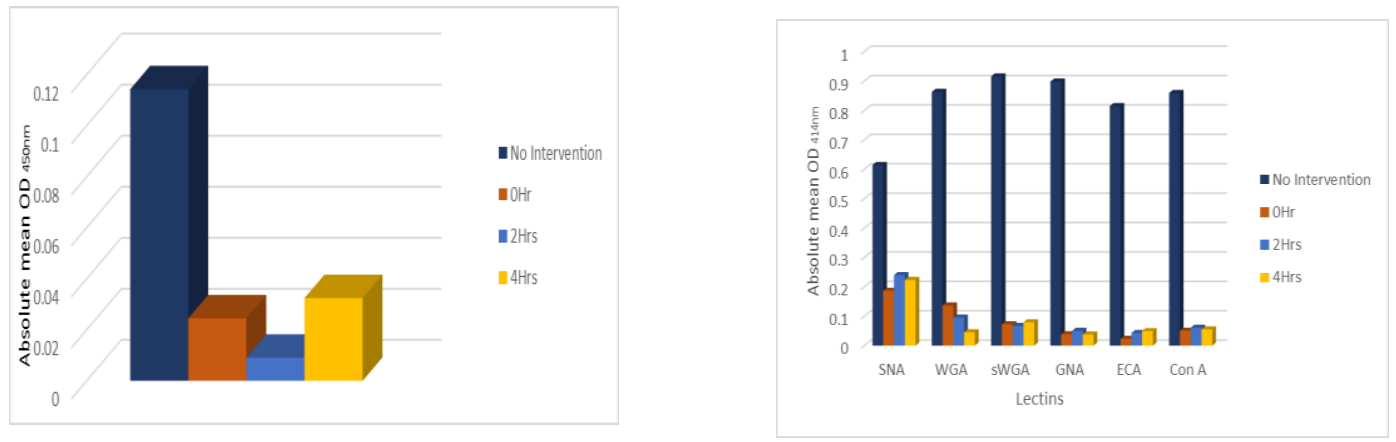

Figure.5 Treatment of biofilm with sialic acid aldolase

The effect of sialic acid aldolase in EPS to prevent biofilm development at different incubation time. (b) The effect of CMPNANA on sialylation at different incubation times with lectin staining.
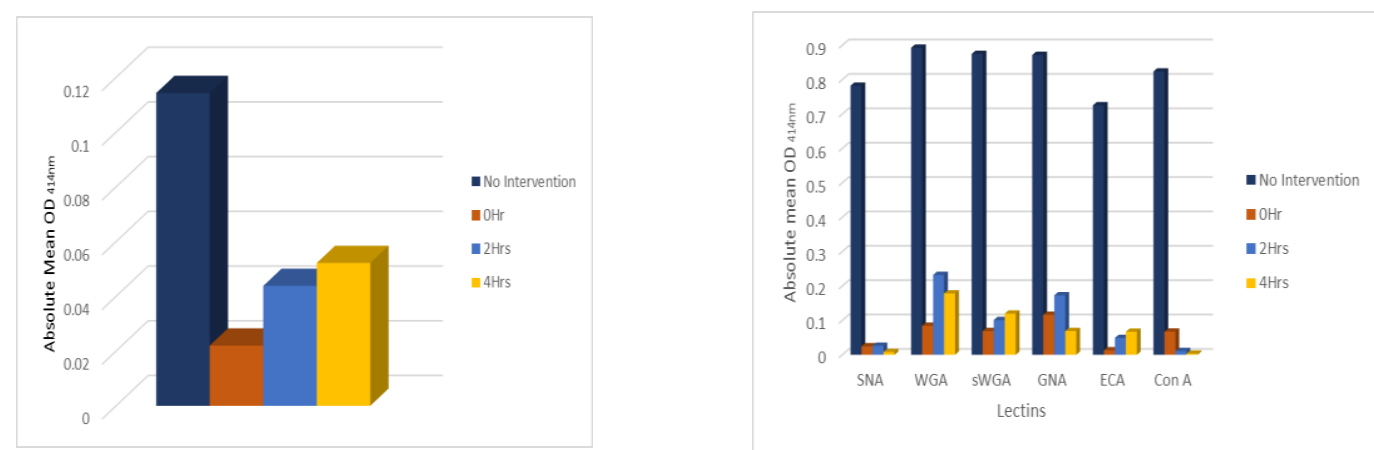

Figure.6 Treatment of biofilm with 1M Hydrochloric acid

(a)The effect of change in $\mathrm{pH}$ in EPS production at different incubation time. (b) Sialic acid $\alpha-2,3 \mathrm{Gal}$, terminal mannose and Gal $\beta-1,4$ GlcNac were observed to be inhibited as $\mathrm{pH}$ changed, however, GlcNAc and high mannose were not inhibited. 


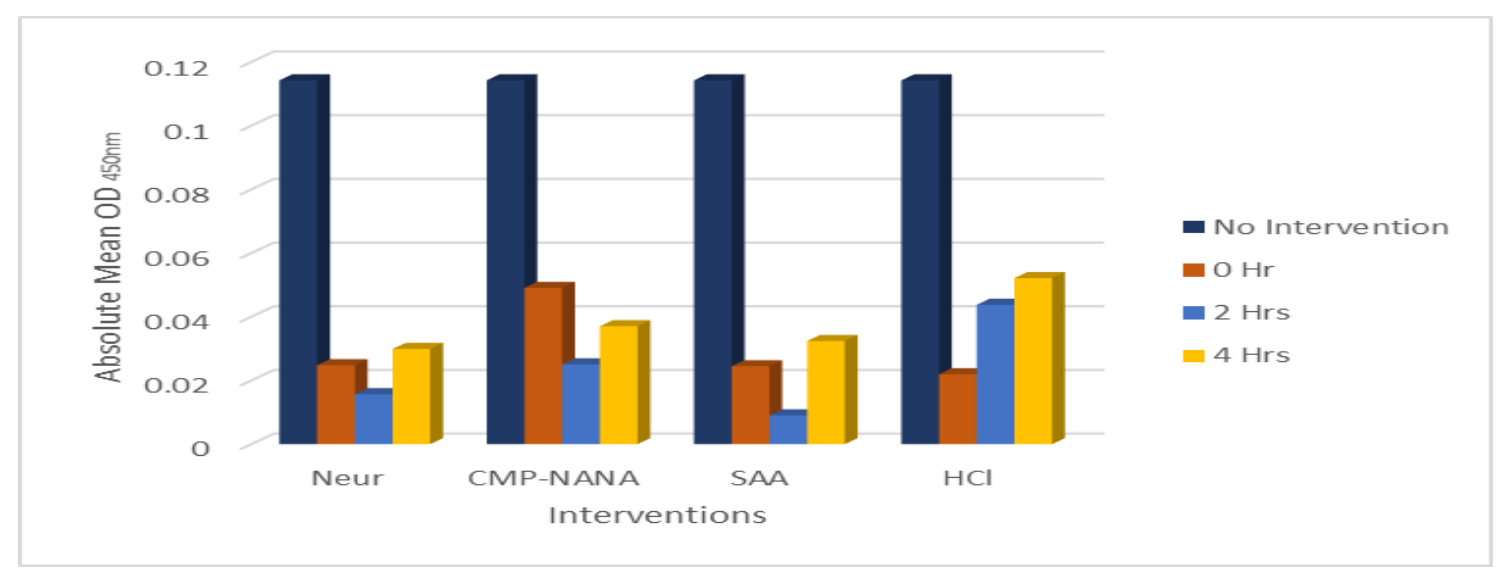

Figure.7 Toluidine blue staining

Neur= Neuraminidase, CMP-NANA= Cytidine 5' - monophospho-N- acetylneuraminic acid Na salt, SAA= Sialic acid aldolase, $\mathrm{HCl}=$ Hydrochloric acid.

The effect of change in $\mathrm{pH}$ on EPS production in $S$. epidermidis biofilm was analysed at different incubation time using toluidine blue stain and is shown in figure $6 \mathrm{a}$. A remarkable overall reduction in EPS production in comparison with the control was observed, however, treatment was most effective at $0 \mathrm{hr}$, relative to $2 \mathrm{hrs}$ and $4 \mathrm{hrs}$. The effect of change in $\mathrm{pH}$ on sialylation of $S$. epidermidis biofilm development at different incubation times was analysed using lectin staining and shown in figure $6 \mathrm{~b}$. It was hypothesised that change in $\mathrm{pH}$ may change the surface charge of the wells thus preventing attachment and possibly prevent biofilm formation. It was observed that the acid remarkably reduced the expression of sialic acid as observed with weak interaction with SNA, however, GlcNAc was most effective at $0 \mathrm{hr}$ as detected by WGA and sWGA. High mannose reduction was observed to be most effective at $4 \mathrm{hrs}$ relative to $0 \mathrm{hr}$ and $2 \mathrm{hrs}$ as detected by GNA. Galactose reduction was observed to be most effective at $0 \mathrm{hr}$ compared to $2 \mathrm{hrs}$ and $4 \mathrm{hrs}$ as detected by ECA and terminal mannose reduction was most effective at $2 \mathrm{hrs}$ and $4 \mathrm{hrs}$ as detected by ConA.

\section{Toluidine blue staining}

The effect of the interventions to prevent biofilm development by intervening with glycosylation at different incubation time is shown in figure 7. A remarkable reduction in EPS production was observed when compared with the control. However, neuraminidase, CMP-NANA and sialic acid aldolase were most effective at $2 \mathrm{hrs}$ of intervention while $\mathrm{HCl}$ was most effective at $0 \mathrm{hr}$ of intervention.

The absolute mean $\mathrm{OD}_{450 \mathrm{~nm}}$ value of $S$. epidermidis biofilm at 24hrs of incubation, $0.248 \pm 0.044 \mathrm{SD}$ indicates that there is biofilm growth, which strongly adheres to the plate surface. This is consistent with previous studies by Deighton et al. (2001), and also confirms that $S$. epidermidis RP62A (ATCC 35984) is a strong biofilm producer. The crystal violet stained cell optical density confirmed the biofilm thickness that is expressed as an index of adherence to the surface of the plate and not the presence of EPS. However, according to Crémet et al. (2013) EPS represent the cell biomass and not its viability.

Decrease in S. epidermidis biofilm growth curve stained with crystal violet corresponds with increase in EPS production which in turn cocooned the cells from staining. Hence, $S$. epidermidis strong adherence ability as earlier reported by (Deighton et al., 2001).

High specificity of lectins for glycan molecule ensures high sensitivity to detect alterations in glycosylation due to malignancies or other immune disease (Rosenfeld et al., 2007). N-acetyl glucosamine was expressed at 30 minutes because it is a major component of EPS in addition to bacterial cell wall (Khan et al., 2013), which was synthesised as the bacteria grow. N-acetyl galactosamine expression may be involved in cellto-cell communication, which is very important in biofilm formation. $\mathrm{N}$-acetyl galactosamine is also 
commonly found in mucins (Varki et al., 2009). Gal $\beta 1,4$ GlcNAc, which is the second or third most abundant glycan in $S$. epidermidis biofilm (Bales et al., 2013), also constitutes the core of blood group carbohydrates that is expressed in abundance in normal cells but reduced in malignant cells (Ghazarian et al., 2011). Mannose is the predominant glycan in $S$. epidermidis biofilm (Bales et al., 2013), and is associated with inflammatory response (Yates 2005). Bales et al. (2013) reported that microorganisms use mannose as component of surface antigens or capsule. Expression of terminal sialic acid was expected because it is found at the cell surface and secreted molecules (Varki, 2007), and first comes in contact with the lectin. Many carbohydrate structures such as terminal sialic acid linked to $\alpha$ 2,3 Gal have been reported to be expressed in various human malignant cells (Shida et al., 2009). Sialic acid has been observed to either act as a biological masking (antirecognition) agent, by shielding recognition sites or as a biological recognition agent for a wide variety of molecules such as lectins and antibodies (Schauer, 2009). ELLA is a useful method for studying qualitative and quantitative variations in the polysaccharides components of a developing biofilm (Leriche et al., 2000).

The remarkable reduction in EPS production at different time of incubation relative to the control shows that neuraminidase reduced sialylation by cleaving sialic acid from the glycoprotein, which is crucial for biofilm formation thereby inhibiting biofilm formation, however, it was most effective at $2 \mathrm{hrs}$, which depicts that treatment should be initiated before $2 \mathrm{hrs}$ of biofilm formation. In comparison of the effect of neuraminidase on sialylation stained with lectins, a remarkable reduction in sialic acid interaction with SNA and WGA at $0 \mathrm{hr}$ and $2 \mathrm{hrs}$ also confirms that neuraminidase desialylated sialic acid from the glycan moieties. Neuraminidase has been reported to cleave cell surface sialic acid (Suzuki and Abe 2014). This study, confirmed that neuraminidase cleaved sialic acid at the early stage of biofilm development, within $2 \mathrm{hrs}$ of biofilm growth. This may be because sialic acid is typically attached to the terminal positions of several glycan molecules. The remarkable increase at $4 \mathrm{hrs}$ compared to $0 \mathrm{hr}$ and $2 \mathrm{hrs}$ was expected because at this time the biofilm has developed and matured therefore treatment with neuraminidase was ineffective. The use of neuraminidase is of medical importance in treating biofilm associated infections at the early stage before biofilm development. Most neuraminidases are observed to cleave specific sialic acid linkages. In this study, neuraminidase from Clostridium perferingens rapidly cleaved $\alpha$ 2,6 linkages. However, neuraminidase from Arthrobacter ureafaciens, exhibits high cleavage potential for $\alpha-2,6$ linkages; while some linkages may be completely or partially resistant to neuraminidase. In contrast, $\alpha-2,3$ linkages are cleaved more rapidly and easily than $\alpha-2,6$ linkages (Varki et al., 2009). This phenomenon may be the reason for the increased expression of sialic acid linked with $\alpha-2,6$ linkage at $4 \mathrm{hrs}$ relative to $0 \mathrm{hr}$ and $2 \mathrm{hrs}$. Also, modification of the sialic acid molecules (methylation, sulfation, succinylation and acetylation) can prevent cleavage (Varki et al., 2009), and modification of glycan molecules enable microorganisms to evade immune defence (Schauer, 2009).

The remarkable decrease in EPS production when treated with CMP-NANA compared to the control depicts that CMP-NANA reduced sialylation. Treatment was most effective at $2 \mathrm{hrs}$ relative to Ohr and 4hrs. Comparing this result with lectin staining in figure 4b, CMP-NANA reduced sialylation at $0 \mathrm{hr}$ as detected by SNA and WGA. It was hypothesised that CMP-NANA is a substrate for sialylation; however, the result obtained was the opposite. CMP-NANA reduction in sialylation caused a remarkable reduction in other sugars like mannose and GlcNAc. Weak interaction of other glycans with their respective lectins suggests that they were either modified by methylation, sulfation, succinylation and acetylation processes or the anomer conformation was changed.

The reduction in EPS production of S. epidermidis biofilm due to treatment with sialic acid aldolase confirms the degradation of sialic acid; and was most effective at $2 \mathrm{hrs}$ of treatment. However, in comparison with lectin screening (figure $5 \mathrm{~b}$ ), it was most effective at $0 \mathrm{hr}$ and a remarkable reduction in sialylation was seen when compared with the control. This result suggests that sialic acid aldolase reversibly degrades sialic acid, which 
confirms previous study, which reported that sialic acid aldolase degrades sialic acid into pyruvate and $\mathrm{N}$ - acetyl mannosamine; ( $\mathrm{Yu}$ et al., 2006). Sialic acid aldolase has been widely used for the chemoenzymatic synthesis of sialic acids and their analogs (Huang et al., 2007).

Alteration of $\mathrm{pH}$ remarkably reduced EPS production and was most effective at $0 \mathrm{hr}$ compared to $2 \mathrm{hrs}$ and $4 \mathrm{hrs}$ of incubation. It was hypothesised that $\mathrm{pH}$ alteration changes the surface charge of the plate which invariably prevents bacterial attachment, which is critical in biofilm formation (Hazan et al., 2006). The lectin staining showed a remarkable reduction in sialic acid expression as sialylation was reduced due to change in $\mathrm{pH}$. This result is consistent with previous study by Varki et al. (2009), which reported that change in $\mathrm{pH}$ by using a stronger acid can destroy sialic acid moieties. However, GlcNAc and high mannose expression were reduced at $\mathrm{Ohr}$ and $4 \mathrm{hrs}$ respectively. High mannose and GlcNAc are important components of the bacterial cell wall. Due to increased resistance of bacterial biofilms to conventional antimicrobial agents and the increasing spread and rise in antibiotic resistant bacterial strains, it is therefore necessary to develop other effective preventative strategies such as incorporating these glycosylation inhibitors in catheters to treat and prevent biofilm associated infections. Sialic acids are critical in biofilm formation, therefore, its detection, modification and cleavage is of significant biomedical importance. The expression of glycans present in $S$. epidermidis biofilms at different incubation time varies; however, future work will use a wider panel of lectins to detect all the glycans expressed and prevent masking of some glycans observed in this study.

\section{References}

Bales, P. M., Renke, E. M., May, S. L., Shen, Y., \& Nelson, D. C. (2013). Purification and Characterization of Biofilm-Associated EPS Exopolysaccharides from ESKAPE Organisms and Other Pathogens. PloS One, 8 (6): 1-8.

Bryers, J. D. (2008). Medical biofilms. Biotechnology and Bioengineering, 100 (1): 1-18.

Chen, S., Laroche, T., Hamelinck, D., Bergsma, D., Brenner, D., Simeone, D. Brand, R., Haab, B. B. (2007). Multiplexed analysis of glycan variation on native proteins captured by antibody microarrays. Nature Methods, 4 (5): 437-444.

Cogen, A. L., Nizet, V., \& Gallo, R. L. (2008). From bench to bedside skin microbiota: a source of disease or defence? British Journal of Dermatology, 158: 442-455.

Crémet, L., Corvec, S., Batard, E., Auger, M., Lopez, I., Pagniez, F., Dauvergne, S. and Caroff, N. (2013). Comparison of three methods to study biofilm formation by clinical strains of Escherichia coli. Diagnostic Microbiology and Infectious Disease, 75 (3): 252-255.

Đapa, T., Dapa, T., Leuzzi, R., Ng, Y. K., Baban, S. T., Adamo, R., Kuehne, S., Scarselli, M., Minton, N., Serruto, D. and Unnikrishnan, M. (2013). Multiple factors modulate biofilm formation by the anaerobic pathogen Clostridium difficile. Journal of Bacteriology, 195 (3): 545-555.

Deighton, M. a, Capstick, J., Domalewski, E., \& van Nguyen, T. (2001). Methods for studying biofilms produced by Staphylococcus epidermidis. Methods in Enzymology, 336 (1996): 177-195.

Ghazarian, H., Idoni, B., \& Oppenheimer, S. B. (2011). A glycobiology review: carbohydrates, lectins and implications in cancer therapeutics. Acta Histochemica, 113(3), 236-47. doi:10.1016/j.acthis.2010.02.004

Hazan, Z., Zumeris, J., Jacob, H., Raskin, H., Kratysh, G., Vishnia, M., ... Lavie, G. (2006). Effective prevention of microbial biofilm formation on medical devices by low-energy surface acoustic waves. Antimicrobial Agents and Chemotherapy, 50(12), 4144-52. doi:10.1128/AAC.00418-06

Hopkins, S., Cookson, B., \& Kessel, A. (2011). English National Point Prevalence Survey on Healthcareassociated Infections and Antimicrobial Use , 2011, 1-140. Retrieved from http://www.hpa.org.uk/

Khan, M. M. T., Takizawa, S., Lewandowski, Z., Habibur Rahman, M., Komatsu, K., Nelson, S. E., ... Ohgaki, S. (2013). Combined effects of EPS and HRT enhanced biofouling on a submerged and hybrid PAC-MF membrane bioreactor. Water Research, 47(2), 747-57. doi:10.1016/j.watres.2012.10.048

Klebensberger, J., Birkenmaier, A., Geffers, R., Kjelleberg, S., \& Philipp, B. (2009). SiaA and SiaD are essential for inducing autoaggregation as a specific response to detergent stress in Pseudomonas aeruginosa. Environmental Microbiology, 11(12), 3073-86. doi:10.1111/j.1462-2920.2009.02012.X

Koo, H., Xiao, J., Klein, M. I., \& Jeon, J. G. (2010). Exopolysaccharides produced by Streptococcus mutans glucosyltransferases modulate the 
establishment of microcolonies within multispecies biofilms. Journal of Bacteriology, 192(12), 3024-32. doi:10.1128/JB.01649-09

Leriche, V., Sibille, P., \& Carpentier, B. (2000). Use of an enzyme-linked lectinsorbent assay to monitor the shift in polysaccharide composition in bacterial biofilms. Applied and Environmental Microbiology, 66(5), 1851-6. Retrieved from http://www.pubmedcentral.nih.gov/articlerender. fcgi?artid=101422\&tool=pmcentrez\&rendertype $=$ abstract

Mack, D., Fischer, W., Krokotsch, a, Leopold, K., Hartmann, R., Egge, H., \& Laufs, R. (1996). The intercellular adhesin involved in biofilm accumulation of Staphylococcus epidermidis is a linear beta-1,6-linked glucosaminoglycan: purification and structural analysis. Journal of Bacteriology, 178(1), 175-83. Retrieved from http://www.pubmedcentral.nih.gov/articlerender. fcgi?artid=177636\&tool=pmcentrez\&rendertype $=$ abstract

Maki, D. G., \& Tambyah, P. a. (2001). Engineering out the risk for infection with urinary catheters. Emerging Infectious Diseases, 7(2), 342-7. doi:10.3201/eid0702.700342

Mody, R., Joshi, S., \& Chaney, W. (1995). Use of lectins as diagnostic and therapeutic tools for cancer. Journal of Pharmacological and Toxicological Methods, 33(1), 1-10. Retrieved from

http://www.ncbi.nlm.nih.gov/pubmed/7727802

Monds, R. D., \& O'Toole, G. a. (2009). The developmental model of microbial biofilms: ten years of a paradigm up for review. Trends in Microbiology, $\quad 17(2), \quad 73-87$. doi:10.1016/j.tim.2008.11.001

Otto, M. (2009). NIH Public Access. Currrent Topic in Microbiology and Immunology, 322, 207-228.

Rosenfeld, R., Bangio, H., Gerwig, G. J., Rosenberg, R., Aloni, R., Cohen, Y., ... Maya, R. B.-Y. (2007). A lectin array-based methodology for the analysis of protein glycosylation. Journal of Biochemical and Biophysical Methods, 70(3), 415-26. doi:10.1016/j.jbbm.2006.09.008
Schauer, R. (2009). Sialic acids as regulators of molecular and cellular interactions. Current Opinion in Structural Biology, 19, 507-514. doi:10.1016/j.sbi.2009.06.003

Sharon, N., \& Lis, H. (2002). How proteins bind carbohydrates: lessons from legume lectins. Journal of Agricultural and Food Chemistry, 50(22), 6586-91. Retrieved from http://www.ncbi.nlm.nih.gov/pubmed/12381156

Shida, K., Misonou, Y., Korekane, H., Seki, Y., Noura, S., Ohue, M., ... Miyamoto, Y. (2009). Unusual accumulation of sulfated glycosphin-golipids in colon cancer cells. Glycobiology, 19(9), 101833. doi:10.1093/glycob/cwp083

Suzuki, O., \& Abe, M. (2014). Galectin-1-mediated cell adhesion, invasion and cell death in human anaplastic large cell lymphoma: regulatory roles of cell surface glycans. International Journal of Oncology, 44(5), 1433-42. doi:10.3892/ijo.2014.2319

Varki, A. (2007). Glycan-based interactions involving vertebrate sialic-acid-recognizing proteins. Nature, 446(7139), 1023-9. doi:10.1038/ nature 05816 .

Varki, A., Schauer, R. (2009). Sialic acids. In: Varki, A., Cummings, R., Esko, J. et al., (eds). Essentials of Glycobiology. 2nd edn. Cold Spring Harbour (NY) Cold Spring Habour Laboratory Press. Available at: http://www.ncbi.nlm.nih.gov/books/NBK1896/ [Accessed August 15, 2014].

Vuong, C., Voyich, J. M., Fischer, E. R., Braughton, K. R., Whitney, A. R., Deleo, F. R., \& Otto, M. (2004). Polysaccharide intercellular adhesin (PIA) protects Staphylococcus epidermidis against major components of the human innate immune system. Cellular Microbiology, 6(3), 269-275. doi:10.1111/j.1462-822.2004.00367.x.

Yu, H., Chokhawala, H. a, Huang, S., \& Chen, X. (2006). One-pot three-enzyme chemoenzymatic approach to the synthesis of sialosides containing natural and non-natural functionalities. Nature Protocols, 1(5), 2485-92. doi:10.1038/nprot.2006.401.

\section{How to cite this article:}

Ekpiken Solomon Ekpiken, Lawrence B. Etim and Mercy Ekong. 2017. Modification of Glycan Expression in Staphylococcus epidermidis to Prevent Biofilm Formation. Int.J.Curr.Microbiol.App.Sci. 6(9): 3604-3613. doi: https://doi.org/10.20546/ijcmas.2017.609.443 